\title{
A BOUQUET-LIKE ATTACHMENT PLATE FOR TELOMERES IN LEPTOTENE OF RYE REVEALED BY HETEROCHROMATIN STAINING*
}

\author{
JULIAN B. THOMAS and PANTOUSES J. KALTSIKES \\ Department of Plant Science, the University of Manitobo, Winnipeg, \\ Manitoba R3T 2N2, Canada
}

Received 25.ii. 75

\begin{abstract}
SUMmaRY
Using a " $\mathrm{C}$ " banding technique the behaviour of the heterochromatic telomeres of rye (Secale cereale L.) was followed throughout the meiotic cycle. Marked aggregation of these telomeres occurred during leptotene concurrently with nucleolar fusion. A conspicuous chromocentre was formed at the nuclear membrane, often in association with the single nucleolus. Telomere groupings at later stages, which derived from this chromocentre, included nonheterochromatic telomeres which indicated that the association did not depend on the presence of heterochromatin.

This aggregation of the telomeres at the nuclear membrane makes leptotene in rye comparable to published reports of the leptotene bouquet, even though characteristic polarisation of the chromosome segments close to the bouquet attachment plate was not seen in rye. Because telomere aggregation is often associated with nucleolar fusion, it is concluded that similar attachments are formed when nucleoli fuse at meiosis of hexaploid wheat, although, because of the absence of heterochromatic telomeres in wheat, no chromocentres are observed.
\end{abstract}

\section{INTRODUGTION}

Formation of the leptotene bouquet is believed to occur when points at which the chromosomes are attached to the nuclear membrane become concentrated on a restricted area of the nuclear membrane (attachment plate of Kaufmann, 1925); in most cases this grouping seems to involve the telomeres (Wilson, 1934). At some point in time the regions of the chromosome adjacent to this attachment plate may become oriented parallel to one another and perpendicular to the attachment plate (Marengo, 1949), or they may acquire a "whirling" appearance around the attachment plate (Hiraoka, 1941).

Synapsis is usually reported to begin in the chromosome regions close to the attachment plate and to spread along the polarised threads toward the interior of the nucleus (Wilson, 1934; Darlington, 1937; Hiraoka, 1941). Since the bouquet is usually recognised from the characteristically polarised chromosomes, it should be borne in mind that there is some degree of polarisation in any interphase nucleus. Because the centromeres are drawn to the pole at anaphase the nuclei begin interphase with the centromeres closely grouped at the polar end of the nucleus and with the telomeres grouped at the other end (Rabl orientation) (Rieger et al., 1968). Thus, there are many instances in which bouquet polarisation does not

* Contribution No. 417 from the Department of Plant Science, University of Manitoba, Winnipeg, R3T 2N2, Canada. 
preclude the possibility that the telomeres do become tightly grouped in early prophase. Among the grasses the telomeres seem to be important in determining the choice of a pairing partner and the patterns of synapsis (Kasha and Burnham, 1965; Michel and Burnham, 1969; Burnham et al., 1972).

Perhaps the grouping of the telomeres is an essential feature of leptotene in these species in spite of the absence of an easily recognised bouquet polarisation.

The species Secale cereale L. (rye) possesses large heterochromatic blocks located on most of the telomeres of its seven pairs of chromosomes. Since a specific stain now exists for this type of heterochromatin (Sarma and Natarajan, 1973) and since the amount of intercalary heterochromatin is small, any clustering of the telomeres into a bouquet-like arrangement should be very easy to detect. Consequently, we examined pollen mother cells (PMG) of rye stained by this method to see if aggregation of the terminal heterochromatin occurred at any point in the meiotic cycle.

\section{Materials AND MEthods}

Inflorescences of Secale cereale L. (cv. Prolific and a bulk population of fall rye) were fixed in Carnoy's II for 1 to 24 hours and then placed in 70 per cent alcohol. Columns of tapetal and pollen mother cells (PMC) from one anther per floret were extruded into acetocarmine to determine the meiotic stage. The other two near synchronous anthers from the floret were then squashed in 45 per cent acetic acid. These latter slides were stained using a standard " $\mathrm{C}$ " type heterochromatin schedule (Thomas and Kaltsikes, 1974). The stain used was either Giemsa or Leishman's.

\section{Results}

The most useful criterion of meiotic sequence lies in the developmental gradients that occur along the spikes of rye. These pass from old in the centre spikelets to young in the spikelets at the base and apex. Anthers in which the archesporium was non-mitotic but the tapetum was highly mitotic were in pre-meiotic interphase (figs. 1 and 2). Sometime after the tapetum was synchronised (the tapetal nuclei became uniform in size and non-mitotic) PMC entered leptotene (figs. 3 and 4). The frequency of nucleoli per PMC fell during leptotene such that by zygotene there was only one nucleolus in each cell. The tapetum in all cases divided synchronously during zygotene. Zygotene (figs. 5 8) was followed by pachytene (figs. 9-11) which was followed by typical diplotene (fig. 12) with no sign of a schizotene stage or a diffuse stage. Consequently the overall sequence of meiotic development in rye (figs. 1-13) was found to agree with that described for hexaploid wheat (Triticum aestivum L.) by Bennett et al. (1973) except for the question of the period of nucleolar fusion. In this respect we agreed with Darvey and co-workers who concluded that nucleolar fusion does occur during leptotene of hexaploid wheat (Darvey, 1972; Darvey et al., 1973). In contrast Bennett (Bennett et al., 1973; Bennett, quoted in Shepard et al., 1974) reported that nucleolar fusion occurs prior to leptotene in hexaploid wheat.

Since the slides stained for heterochromatin were pre-classified for their 
meiotic stage, cyclic change in the distribution of the telomeric heterochromatin was assessed without bias. During the pre-meiotic interphase the terminal " $\mathrm{G}$ " bands were either distributed in one half of the nucleus in association with the nucleoli (fig. 1), or were distributed right across the flattened nucleus (fig. 2). These two arrangements were probably equatorial and polar views of a persistent $\mathrm{Rabl}$ orientation established at anaphase of the last pre-meiotic mitosis. In many cells a dark staining diffuse area was also noted in addition to the intensely staining and condensed telomeres. Where the telomeres were distributed in one half of the nucleus this dark staining region was restricted to the opposite half (fig. 1). This bipolar appearance was also noted in PMG in early meiotic prophase (compare

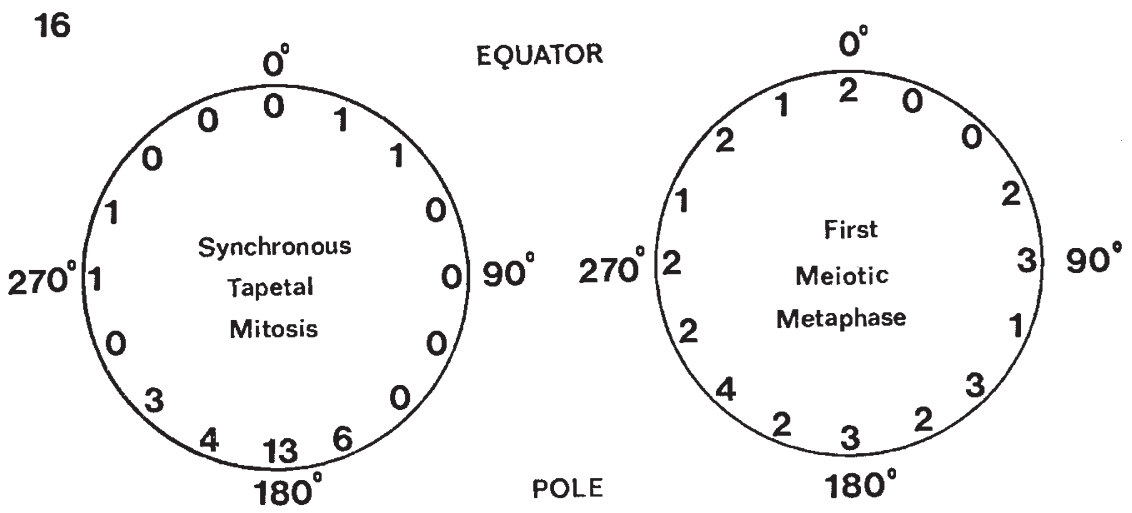

Fig. 16.-The circular distribution of nuclear orientations in binucleate tapetal cells at two different stages of development. (Nuclear orientation represents the angle of the dark staining diffuse area about the nuclear centre. Estimates to the nearest sixteenth of a clockwise revolution were made from the intersection of the nuclear margin with the axis joining the two nuclear centres. These data were obtained from the photomicrographs in figs. 14 and 15 by an independent observer after the photos were coded and randomised.)

fig. 1 with figs. 3 and 4), and in interphase tapetal cells (figs. 14 and 15) and in somatic cells generally. Since in all these cases the dark staining diffuse region of the nucleus was found to lie opposite the interphase telomeres we believe that it represents that part of the nucleus that was formed from the polar area. Anaphase convergence of the proximal regions into the pole would result in a high density of euchromatin around the centromeres. The orientation of sister nuclei within the binucleate tapetal cells also suggested that the interphase centromeres were located in this dark staining diffuse area. Immediately following the synchronous tapetal mitosis most nuclei were arranged with their telomere region facing the sister nucleus, and with the dark staining diffuse area orientated away from their sister nucleus and toward the pole position (fig. 14). The circular distribution of nuclear orientations within the binucleate tapetal cells shows that just after the synchronous tapetal mitosis the dark staining diffuse area lay almost exclusively within the polar quadrant (fig. 16). Strong association between the pole of the cell and this area of the nucleus immediately following anaphase confirms the idea that it indicates the position of the centromeres within the interphase nucleus. Later on (by first meiotic metaphase) the 
orientation of this "centromere area" was nearly random relative to the location of the sister nucleus (figs. 15 and 16). Since the anaphase orientation of the nucleus was not conserved some rotation of the nucleus probably occurred during interphase.

As the PMC passed through the beginning of meiotic prophase there was marked aggregation of the heterochromatic telomeres in the same area of the nucleus that was occupied by the telomeres in interphase (figs. 3 and 4). This aggregation resulted in the formation of a single large chromocentre and was accompanied by progressive fusion of the nucleoli so that in the end each PMC exhibited one very prominent heterochromatic body, often closely associated with the single nucleolus and located at the nuclear margin (figs. 5 and 6). Although association between the nucleolus and the chromocentre was noted in most cells it was not present in all (fig. 5). Injection of a 0.1 per cent solution of colchicine into the boot three days before fixation blocked the formation of the chromocentre in rye. The chromocentre persisted well into the period of chromosome synapsis (fig. 7) but later on it began to break up before the beginning of pachytene (figs. 8-12). However telomere groupings were found in many pachytene nuclei (figs. 9 and 10) and in diplotene most nuclei showed close end-to-end associations between two or more bivalents (fig. 12). Non-heterochromatic telomeres were found both in telomere groups at pachytene (fig. 10) and in the end-to-end associations of diplotene. Statistical analysis of 26 end-to-end associations of two, three or four telomeres at diplotene, in a plant which exhibited 10 heterochromatic telomeres, showed that heterochromatic and non-heterochromatic telomeres participated in these associations as expected on the basis of 10 heterochromatic telomeres to four non-heterochromatic. Therefore it is likely that non-heterochromatic telomeres were also included in the chromocentre that was formed earlier.

\section{Discussion}

\section{(i) The attachment plates in rye and wheat}

Heterochromatin staining reveals that during early meiotic prophase the telomeres of rye chromosomes acquire a remarkable property that results in their becoming tightly grouped at the nuclear membrane. It could be argued that rye is a special case and that the chromocentre is an example of non-specific groupings of heterochromatic loci (Walters, 1970). However, the presence of non-heterochromatic telomeres in the telomere groupings of pachytene and diplotene suggest that it was indeed telomeres which aggregated and not merely the associated heterochromatin. This tight grouping of the telomeres at leptotene is comparable to the attachment plate in other, more obvious bouquets. On the other hand the typical bouquet appearance with parallel or spiral arrangement of the near terminal chromosome segments was not seen in rye. Consequently, without the terminal heterochromatin as a marker for the chromosome ends the grouping of the telomeres might well have gone unnoticed. Since the chromosomes of wheat lack this pronounced heterochromatin it is interesting to note that some parallels exist between the cryptic bouquet in rye and the process of nucleolar fusion in wheat.

In hexaploid wheat there are two pairs (1B and $6 \mathrm{~B}$ ) of conspicuously 
satellited chromosomes (Gill et al., 1963) which are the most active among the nucleolus organising chromosomes (Crosby, 1957; Longwell and Svihla, 1960); their nucleolus organising regions (NOR's) are probably exclusively responsible for the maximum of four large nucleoli that is usually found in wheat (Darvey and Driscoll, 1972).

In the course of meiosis the mean number of nucleoli per cell is reduced to one before or during leptotene (Darvey, 1972; Bennett et al., 1973; Darvey et al., 1973). This common nucleolus lies adjacent to the nuclear margin (Bennett et al., 1973). Subsequently two bivalents are generally seen attached to the single nucleolus at diakinesis (Darvey, 1972) which shows that during leptotene all four NOR's are brought to a common region of the nucleus, adjacent to the nuclear membrane. Secondary constrictions are close to the shortarm telomeres in both wheat (Gill et al., 1963) and rye (Heneen, 1962) so perhaps the short-arm telomeres have a role in nucleolar fusion. Strong association between the fused rye nucleolus and the telomere attachment plate was noted earlier. It is plain that the strange behaviour of wheat nucleolus organisers, whereby both homologous and non-homologous nucleoli all fuse together, can be explained if we assume that wheat chromosomes participate in an attachment plate similar to that noted in rye. Inclusion of the telomeres on $1 \mathrm{~B}^{\mathrm{s}}$ and $6 \mathrm{~B}^{\mathrm{s}}$ in such a bouquet could bring all four NOR's sufficiently close together to guarantee complete nucleolar fusion close to the nuclear membrane.

\section{(ii) Chromosome association and the bouquet}

What could be the significance of the leptotene attachment plate in rye. One obvious possibility is that it represents a process whereby homologous chromosomes locate one another prior to detailed synapsis. Since chromosome association in wheat $\times$ triticale (unpublished) and formation of the bouquet (in rye) are both inhibited by colchicine and since both occur in the same general period of meiosis they seem to be different aspects of the same process. Formation of the bouquet seems to be achieved by an organising centre (pole determinant) in the cytoplasm of the PMC adjacent to the tapetum. The same organising centre may also be concerned with the control of pore development. Dover (1973) noted several lines of evidence that indicated a connection between pore abnormalities and unusual pairing conditions in the cereals. High levels of homoeologous pairing were associated with multi-pore pollen whereas colchicine induced asynapsis was associated with poreless pollen. Formation of the pore in Sorghum, another grass (Christensen and Horner, 1974) occurred at the four points of contact between the quartet and the inner wall of the tapetum. This is the same region of the cytoplasm which was associated with the formation of the heterochromatic aggregate. These considerations suggest that the control of chromosome pairing is integrated into a much wider scheme of developmental control which also regulates the areas of meiotic division and the normal development of pollen (Dover, 1972, 1973). It also seems likely that this overall control is regulated by a colchicine sensitive organising centre located in the PMC between the nucleus and the tapetum. Whereas Dover concluded that pore formation and chromosome pairing were both inhibited by colchicine in $G_{1}$ (Dover, 1972; Dover and Riley, 1973), our data as well as those of other authors (Dermen, 1938; Walker, 1938; Levan, 1939; Barber, 1942; 
Darvey, 1972; Shepard et al., 1974) indicate that colchicine can act on pairing, and possibly pollen pore formation, as late as $G_{2} /$ leptotene.

\section{(iii) Formation of the attachment plate}

With these ideas in mind it is worth pointing out some features that have been reported concerning both the telomeres and the formation of the leptotene bouquet. In the presence of a leptotene bouquet in both Psilotum (Hiraoka, 1941) and Onoclea (Marengo, 1949) the entire leptotene nucleus was displaced toward the cell margin on the side of the attachment plate. Thus there seemed to be some mechanical interaction between nucleus and cytoplasm associated with the bouquet. In general, where there is a centriole present in the cytoplasm it is found close to the attachment plate of the bouquet (Wilson, 1934; see esp. Hughes-Schrader, 1943). Hughes-Schrader (1943) reported a series of changes in astral activity around the centriole that correlated with the polarisation of chromosomes within the prophase nucleus. Initially the attachment plate of the leptotene bouquet was formed close by an active centriole complex (aster) in the cytoplasm, but after the aster disappeared in mid-prophase, bouquet polarisation was lost. However, when the asters reappeared in diplotene the telomeres regained their ability to polarise the bivalents toward the aster. Finally polarisation by the telomeres lapsed about the time that the nuclear membrane was dissipated (Diakinesis). At the same time the centromeres became active and began to orient the bivalents for meiotic metaphase. These results suggest that during the meiotic cycle the telomeres might have the ability to interact with a spindle-like system in the cytoplasm but only through association with the nuclear membrane. In fact centromere-like activity (neocentric activity) of particular telomeres has also been found in the meiosis of rye. In certain inbred lines of rye these telomeres are able to polarise part or all of the chromosomes to one pole or the other, but only during the two meiotic divisions (Prakken and Muntzing, 1942; Rees, 1955). Incomplete removal of membrane material from the telomeres after meiotic prophase could allow the telomeres to retain properties normally concerned with formation of the bouquet attachment plate, and to behave like single centromeres on the meiotic spindle (Ostergren and Prakken, 1946).

Bennett et al. (1974) reported that bundles of $20 \mathrm{~nm}$ fibres appear within the PMC nucleus during pre-meiotic interphase of hexaploid wheat. Some of these bundles were found in the narrow gaps between the chromosomes and the nuclear membrane. The authors suggested that this material could be a specific structure involved in meiotic interaction between the nuclear membrane and the chromosomes.

\section{(iv) Other meiotic systems}

In summary, detection of a cryptic bouquet stage in rye and its inferred existence in wheat suggests that bouquet arrangements may be more widespread than has been suspected hitherto. It is possible that the formation of an attachment plate in leptotene corresponds directly to the colchicine sensitive pairing activity that has been detected in wheat.

However, despite the emphasis that we have placed on telomeres in the present discussion, in different meiotic systems other parts of the chromosome could be drawn to the attachment plate. Furthermore, since colchicine 
sensitive activity is not essential for synapsis provided chromosome segments are close enough to one another (Driscoll et al., 1967; Driscoll and Darvey, 1970) it follows that a leptotene attachment plate might be redundant in species exhibiting intense somatic association. These simple considerations may explain much of the divergence between different accounts of meiosis (cf. Wenrich, 1917; McClung, 1927).

Acknoroledgments.-Financial assistance from the International Development Research Centre and the National Research Council of Canada, both of Ottawa, is gratefully acknowledged. We would also like to thank Dr Norman Darvey for discussing with us the ideas in this paper, and Mr Graham Scoles for scoring the data in fig. 16.

\section{REFERENCES}

BARBER, H. N. 1942. The experimental control of chromosome pairing in Fritillaria. $\mathcal{F}$. Genet., 43, 359-374.

BENNETT, M. D., RAO, M. K., SMITH, J. B., AND BAYLISS, M. W. 1973. Cell development in the anther, the ovule and the young seed of Triticum aestivum L. var. Chinese Spring. Phil. Trans. Roy. Soc. B., 266, 39-81.

BENNETT, M. D., STERN, H., AND WOODWARD, M. 1974. Chromatin attachment to nuclear membrane of wheat pollen mother cells. Nature, 252, 395-396.

BURNHAM, C. R., STOUT, J. T., WEINHEIMER, W. H., KNOWLES, R. V., AND PHILLIPS, R. L. 1972. Chromosome pairing in maize. Genetics, 71, 111-126.

CHRISTENSEN, J. E., AND HORNER, H. T. 1974. Pollen pore development and its spatial orientation during microsporogenesis in the grass Sorghum bicolor. Am. F. Bot., 61, 604-623.

CROsBY, A. R. 1957. Nucleolar activity of lagging chromosomes in wheat. Am. F. Bot., $44,813-822$.

Darlington, C. D. 1937. Recent Advances in Cytology, 2nd. Ed. Churchill, London.

DARVEY, N. L. 1972. Studies on spatial relationships of the chromosomes of hexaploid wheat. Ph.D. Thesis, University of New South Wales.

DARVEY, N. L., AND DRISCOLL, C. J. 1972. Nucleolar behaviour in Triticum. Chromosoma (Berl.), 36, 131-139.

DARVEY, N. L., DRISCOLL, C. J., AND KALTSIKES, P. J. 1973. Evidence against somatic and premeiotic association in wheat. (Abstract) Genetics, 74, June Suppl. No. 2, part 2, s57-s58.

DERMEN, H. 1938. Cytological analysis of polyploidy induced by colchicine and by extremes of temperature. 3 . Hered., 29, 211-229.

DOVER, G. A. 1972. The organization and polarity of pollen mother cells of Triticum aestivum. 7. Cell Sci., 11, 689-711.

DOVER, G. A. 1973. On pores and pairing. In Chromosomes Today (ed. J. Wohrman and K. R. Lewis), vol. 4, 197-204. John Wiley, New York.

DOVER, G. A., AND RILEY, R. 1973. The effect of spindle inhibitors applied before meiosis on meiotic chromosome pairing. 7. Cell Sci., 12, 143-161.

DRISCOLL, C. J., AND DARVEY, N. L. 1970. Chromosome pairing: effect of colchicine on an isochromosome. Science, 169, 290-291.

DRISCOLl, C. J., DARVEy, N. L., AND BARBER, H. N. 1967. The effect of colchicine on the meiosis of hexaploid wheat. Nature, 216, 687-688.

GILL, B. S., MORRIS, R., SCHMIDT, J. W., AND MAAN, S. s. 1963. Meiotic studies of chromosome morphology in the Wichita winter wheat variety by means of monosomics. Can. $\mathcal{7}$. Genet. Cytol., 5, 326-337.

HENEEN, w. K. 1962. Chromosome morphology in inbred rye. Hereditas, 48, 182-200.

HIRAOKA, T. 1941. Studies of mitosis and meiosis in comparison. IV. A contribution to the study of the origin of the bouquet and its formation. Caryologia, 11, 483-492.

HUGHES-SCHRADER, s. 1943. Polarisation, kinetochore movements and bivalent structure in the meiosis of male mantids. Biol. Bull., 85, 265-300.

KASHA, K. J. AND BURNHAM, C. R. 1965. The location of interchange break-points in barley. II. Chromosome pairing and the intercross method. Can. F. Genet. Cytol., 7, 620-632.

KAUFMANN, B. P. 1925. The existence of double spiral chromatin bands and a bouquet stage in Tradescantia pilosa Lehm. Am. Nat., 59, 190. 
LEVAN, A. 1939. The effect of colchicine on meiosis in Allium. Hereditas, 25, 9-26.

LONGWEll, A., AND SVirla, G. 1960. Specific chromosomal control of the nucleolus and of the cytoplasm in wheat. Exp. Cell Res., 20, 294-312.

MCCLUNG, C. E. 1927. Synapsis and related phenomena in Mecostethus and Leptysma (Orthoptera). F. Morph. Physiol., 43, 181-252.

MARENGO, N. P. 1949. A study of the cytoplasmic inclusions during sporogenesis in Onoclea sensibilis. Am. F. Bot., 36, 603-613.

MICHEL, K. E., AND BURNHAM, C. R. 1969. The behaviour of non-homologous univalents in double trisomics of maize. Genetics, 63, 851-864.

OSTERgREN, G., AND PRAKKEN, R. 1946. Behaviour on the spindle of the actively mobile chromosome ends of rye. Hereditas, 32, 473-494.

PRAKKEN, R., AND MUNTZING, A. 1942. A meiotic peculiarity in rye simulating a terminal centromere. Hederitas, 28, 442-482.

REES, H. 1955. Genotypic control of chromosome behaviour in rye. I. Inbred lines. Heredity, 9, 93-116.

RIEGER, R., MICHEALIS, A., AND GREEN, M. M. 1968. A Glossary of Genetics and CytogeneticsClassical and Molecular. Springer-Verlag, Heidelberg.

SARMA, N. P., AND NATARAJAN, A. T. 1973. Identification of heterochromatic regions in the chromosomes of rye. Hereditas, 74, 233-238.

SHEPARD, J., BOOTHROYD, E. R., AND STERN, H. 1974. The effect of colchicine on synapsis and chiasma formation in microsporocytes of Lilium. Chromosoma (Berl.), 44, 423-437.

THOMAS, J. B., AND KALTSIKEs, P. J. 1974. A possible effect of heterochromatin on chromosome pairing. Proc. Nat. Acad. Sci. (USA), 71, 2787-2790.

WALKER, R. I. 1938. The effect of colchicine on microspore mother cells and microspores of Tradescantia paludosa. Am. F. Bot., 25, 280-285.

WALTERs, M. s. 1970. Evidence on the time of chromosome pairing from the preleptotene spiral stage in Lilium longiflorum "Croft". Chromosoma (Berl.), 29, 375-418.

WENRIGH, D. H. 1917. Synapsis and chromosome organization in Chorthippus (Stenobothrus) curtipennis and Trimcropteris suffusa (Orthoptera). F. Morph., 29.

wirson, E. B. 1934. The Cell in Devel.ppment and Heredity, 3rd. Ed. Macmillan, New York. 


\section{Plate I}

Figs. 1 and 2-PMC, pre-meiotic interphase (bar on fig. 1 is $25 \mu \mathrm{m}$ ). Figs. 3 and $4 .-$ PMC, leptotene/nucleolar fusion. Figs. 5 and 6.-PMC, early zygotene (fig. 5: arrows indicate chromocentres (stained) and nucleoli (unstained); bar is $50 \mu \mathrm{m}$. Fig. 6: bar is $200 \mu \mathrm{m}$ ). (Figs. 1-5 are fall rye bulk, stained with Giemsa; fig. 6 is Prolific, stained with Leishman's. Figs. 2-4 are same magnification as fig. 1.)

\section{Plate II}

Frg. 7.-PMC, mid zygotene. Frg. 8.-PMC, late zygotene. Fig. 9.-PMC, early pachytene. FIG. 10.-PMC, mid pachytene (arrows with open heads indicate nonheterochromatic telomeres, arrows with solid heads indicate heterochromatic telomeres). Frg. 11.-PMC, late pachytene. FIG. 12.-PMC, diplotene (this cell contains five ring bivalents with a band on both ends and two ring bivalents with a band on one end only). FIG. 13.-PMC, first meiotic metaphase (this cell contains five ring bivalents of which three have a band on one side only and two have a band on both sides; of the two rod bivalents, one has bands on both the paired and unpaired arms and the other has a band only on the unpaired arm." This latter band shows clear heteromorphism between the homologues, the top chromosome carries no prominent heterochromatin at all (arrowed)). (Figs. 7 and 12 are Prolific, rest are fall rye bulk; fig. 13 is stained with Leishman's and has the same magnification as fig. 5 (plate I); rest are stained with Giemsa and have same magnification as fig. 1.)

\section{Plate III}

FIG. 14.-Random sample of interphase binucleate tapetal cells from an anther still containing cells in the synchronous tapetal division. (Bar is $20 \mu \mathrm{m}$ ). Frg. 15.-Random sample of binucleate tapetal cells from an anther in first meiotic metaphase. (Magn. same as fig. 14. This anther was probably 24-36 hours later in development than that illustrated in fig. 14. Both anthers were from the same spikelet of fall rye and both are stained with Giemsa.) 

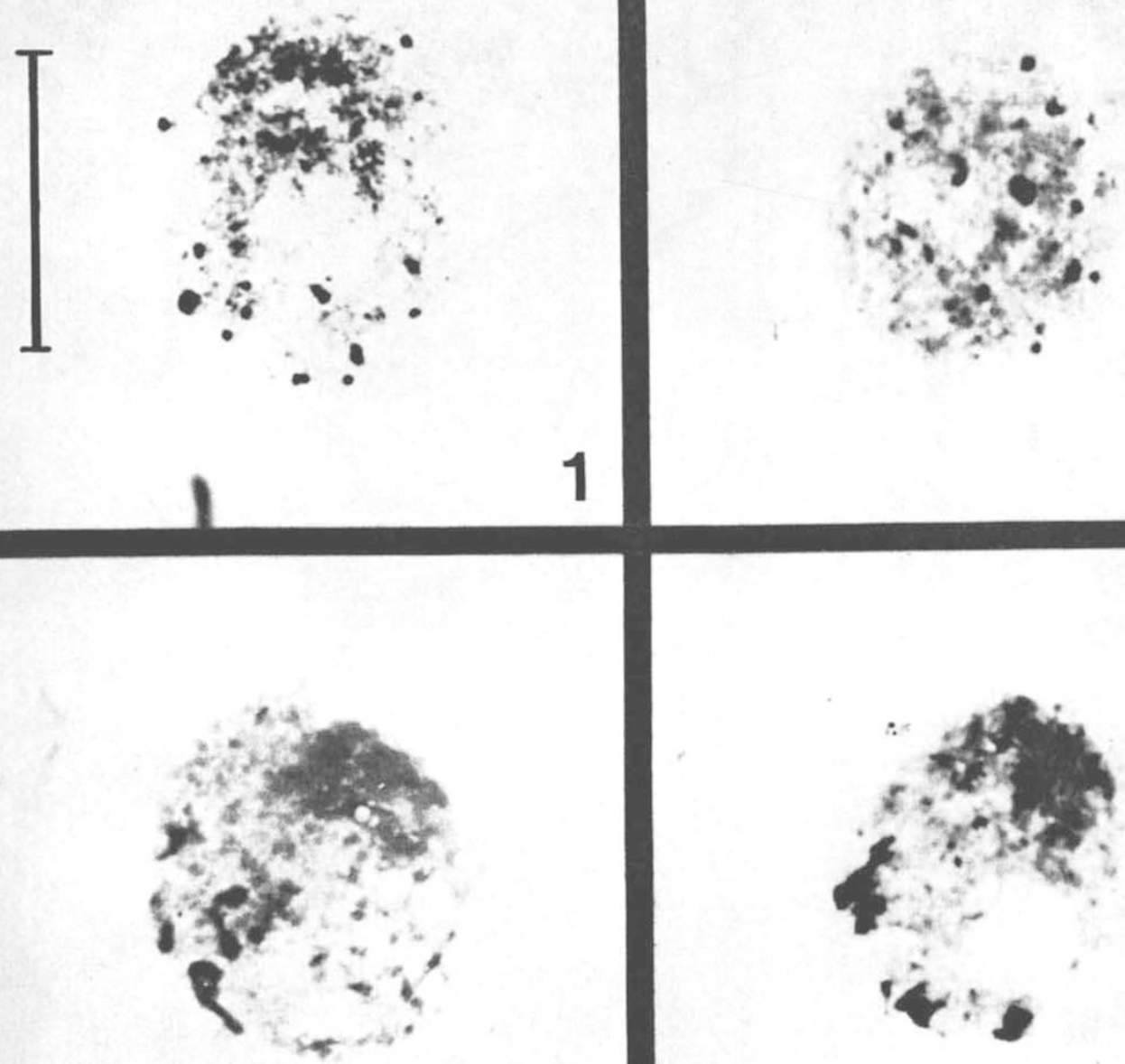

3

4

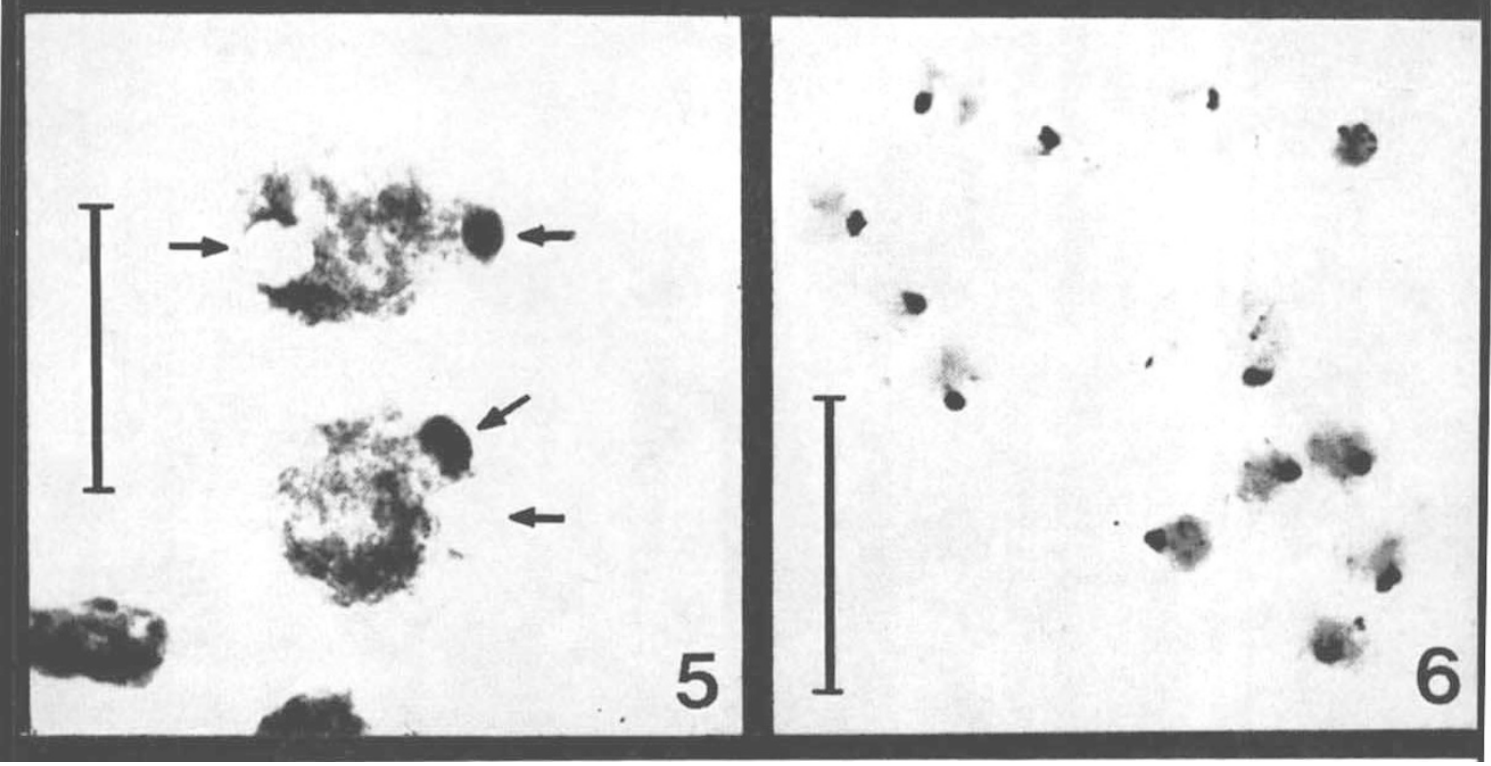



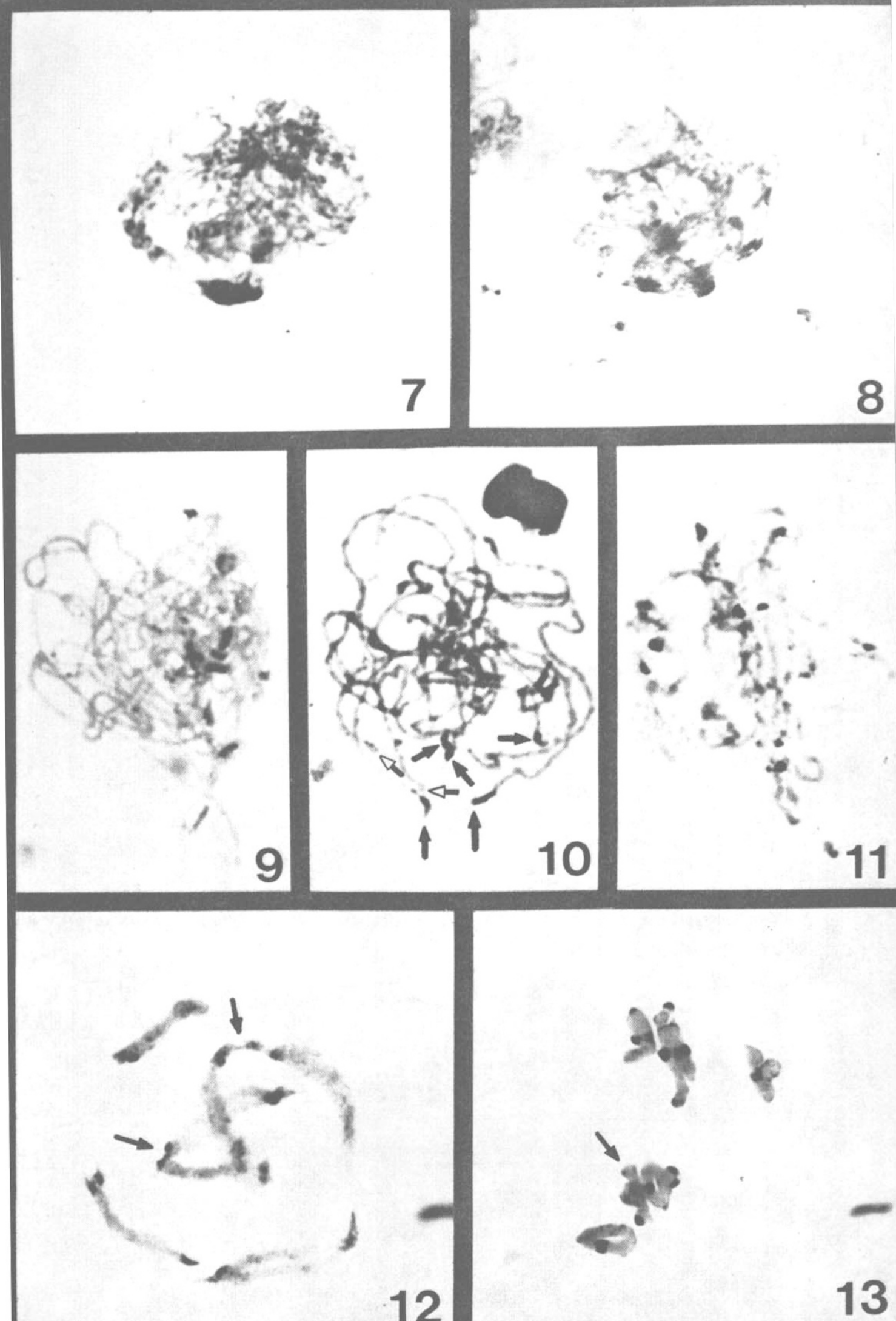

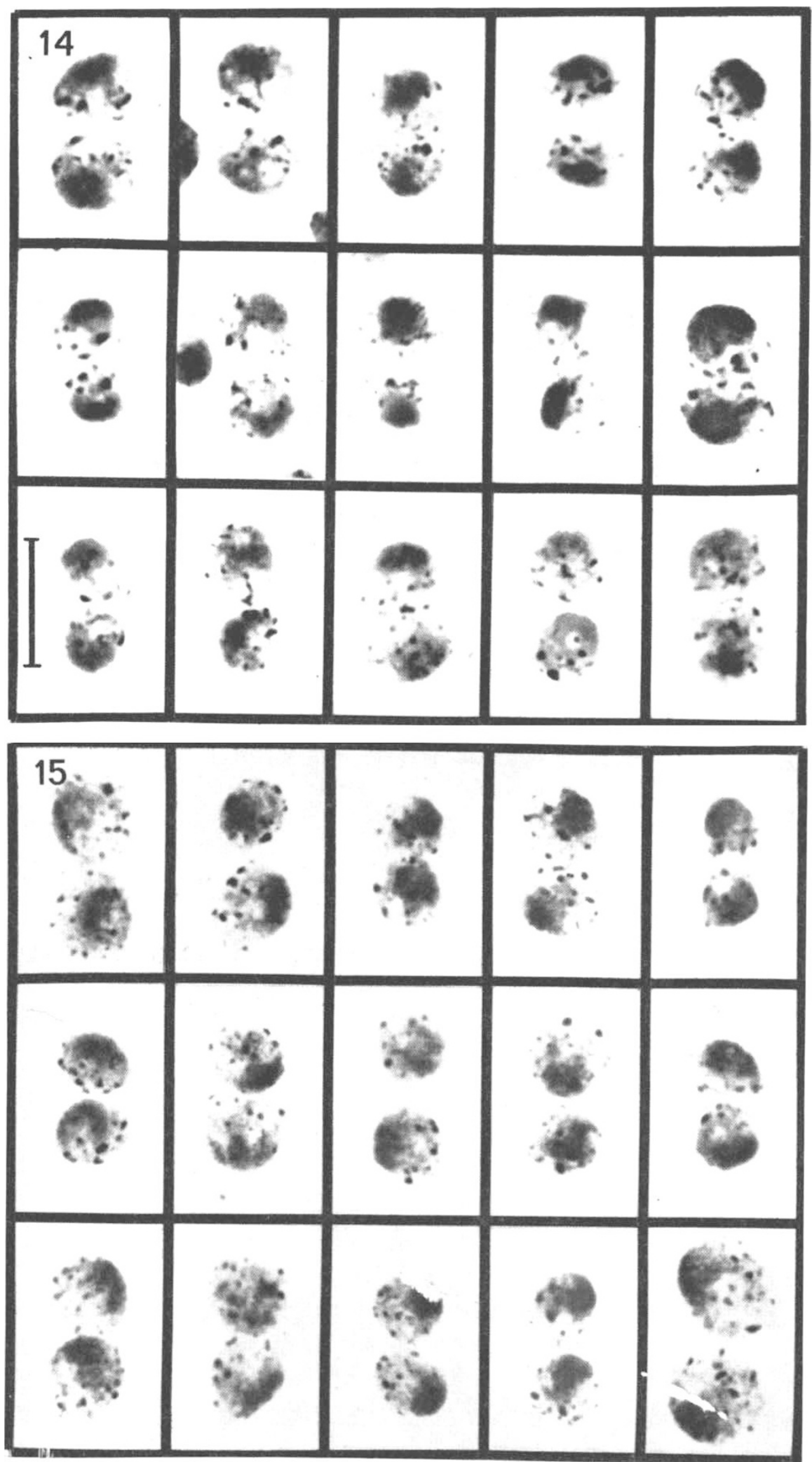